\title{
RETRACTED ARTICLE: Intraperitoneal abscess formation spreading to the liver after endoscopic ultrasound-guided fine-needle aspiration for abdominal lymphadenopathy
}

\author{
Koji Takahashi ${ }^{1}$ [ $\cdot$ Hiroshi Kashimura ${ }^{1} \cdot$ Naoaki Konno ${ }^{1} \cdot$ Miyuki Nakagawa $^{1} \cdot$ Akari Munakata $^{1} \cdot$ Ken Okawara $^{1}$. \\ Yohei Aoki ${ }^{1} \cdot$ Takeshi Nihei $^{1}$
}

Received: 10 October 2018 / Accepted: 4 November 2018 / Published online: 16 November 2018

(c) Japanese Society of Gastroenterology 2018

This article has been retracted by the authors as they did not obtain consent from the patient to publish the case and accompanying images. The article content is no longer available online in order to protect patient confidentiality. All authors agree to this retraction. 